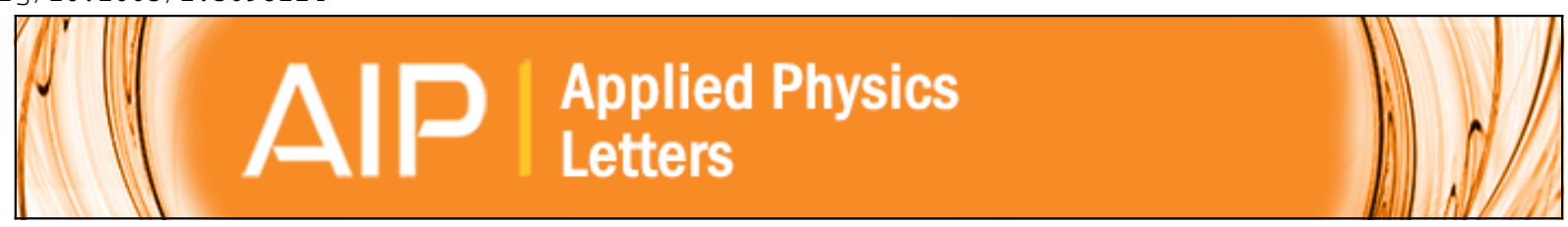

\title{
Giant converse magnetoelectric effect in multi-push-pull mode
}

\section{Metglas/Pb(Zr,Ti)O3/Metglas laminates}

Menghui Li, Yaojin Wang, Davresh Hasanyan, Jiefang Li, and D. Viehland

Citation: Applied Physics Letters 100, 132904 (2012); doi: 10.1063/1.3698114

View online: http://dx.doi.org/10.1063/1.3698114

View Table of Contents: http://scitation.aip.org/content/aip/journal/apl/100/13?ver=pdfcov

Published by the AIP Publishing

\section{AlP Re-register for Table of Content Alerts}




\title{
Giant converse magnetoelectric effect in multi-push-pull mode Metglas/Pb(Zr,Ti) ${ }_{3} /$ Metglas laminates
}

\author{
Menghui Li, ${ }^{\text {a) }}$ Yaojin Wang, Davresh Hasanyan, Jiefang Li, and D. Viehland \\ Department of Materials Science and Engineering, Virginia Tech, Blacksburg, Virginia 24061, USA
}

(Received 3 February 2012; accepted 11 March 2012; published online 27 March 2012)

The converse magnetoelectric (CME) effect was investigated theoretically and experimentally for multi-push-pull mode Metglas $/ \mathrm{Pb}(\mathrm{Zr}, \mathrm{Ti}) \mathrm{O}_{3} /$ Metglas laminates. The experimental and theoretical values of the CME coefficient $\left(\alpha_{\mathrm{B}}\right)$ exhibited similar trends. A large $\alpha_{\mathrm{B}}=6.94 \mathrm{G} / \mathrm{V}$ was observed at $1 \mathrm{kHz}$ under a dc magnetic bias of $11 \mathrm{Oe}$. At an electromechanical resonance frequency of $29.6 \mathrm{kHz}$, the laminate exhibited a giant value of $\alpha_{\mathrm{B}}=79.5 \mathrm{G} / \mathrm{V}$. These results show significantly enhanced CME effects in multi-push-pull mode laminates, compared to previously reported ones with different structures and materials. (c) 2012 American Institute of Physics. [http://dx.doi.org/10.1063/1.3698114]

Magnetoelectric (ME) multiferroics, with coexistence of ferromagnetism and ferroelectricity, have been of recent interest due to their potential applications in multifunctional devices. ${ }^{1-4}$ The ME effects, which are defined as a change in polarization by an applied magnetic field [i.e., direct magnetoelectric (DME) effect] or the induction of magnetization by an applied electric field [i.e., converse magnetoelectric (CME) effect], have been investigated in single-phase and two-phase multiferroics. ${ }^{1-7}$ It has been shown that the DME coefficient $\alpha_{\mathrm{DME}}$ for magnetostrictive/piezoelectric laminate composites are higher than single-phase material or composites with other interphase interconnectivities. ${ }^{6-9}$ These laminates hold promises for many applications, such as magnetic field sensors and actuators.

Laminate composites of $\mathrm{Pb}\left(\mathrm{Mg}_{1 / 3} \mathrm{Nb}_{2 / 3}\right) \mathrm{O}_{3}-\mathrm{PbTiO}_{3}$ (PMN-PT) and Terfenol-D, or PMN-PT and Metglas have been reported to exhibit large CME effects at low frequency under a small dc magnetic bias $\left(\mathrm{H}_{\text {bias }}\right){ }^{10-12}$ In these papers, the effect of thickness of the magnetostrictive phase was not considered, which is known to remarkably affect the ME properties. ${ }^{13-15}$ These prior studies were for laminates longitudinally magnetized and transversely poled: termed as a L-T mode. Moreover, the ME effects in longitudinally magnetized and longitudinally poled, or (L-L) mode, laminates are notably larger than the L-T ones, which is mainly due to the longitudinally piezoelectric coefficients $\left({ }^{p} d_{33}\right)$ of PMN$\mathrm{PT}$ or $\mathrm{Pb}(\mathrm{Zr}, \mathrm{Ti}) \mathrm{O}_{3}$ (PZT) being larger than the transverse one $\left({ }^{p} d_{31}\right) .{ }^{6-9}$ However, few reports have been made concerning the CME effect.

In this paper, we investigated the CME effects for multipush-pull mode Metglas/PZT/Metglas laminates. A theoretical model is presented that predicts large CME coefficients $\left(\alpha_{\mathrm{B}}\right)$. Experimental results reveal good agreement with the predicted ones. In particular, using an optimum thickness ratio, giant CME effects have been found, which exhibit a further enhancement at the resonance frequency.

This investigation considers the multi-push-pull mode Metglas/PZT/Metglas sandwich structure in the $(2,3)$ plane.

\footnotetext{
${ }^{\text {a) }}$ Author to whom correspondence should be addressed. Electronic mail: limh09@vt.edu.
}

We assumed that the polarization of the piezo-fibers was uniformly arranged along the longitudinal direction (3-axis). Thus, the multi-push-pull configuration can be considered as a multi-L-L mode, as illustrated in Fig. 1. Furthermore, we only considered the stresses in the 3-direction.

An averaging method is used for deriving the effective material parameters of the composite. The piezoelectric constitutive equations can be written as

$$
{ }^{p} S_{3}={ }^{p} S_{33}{ }^{p} T_{3}+{ }^{p} d_{33} E_{3}, \quad D_{3}={ }^{p} d_{33}{ }^{p} T_{3}+{ }^{p} \varepsilon_{33} E_{3},
$$

where ${ }^{m} S_{3}$ and ${ }^{p} T_{3}$ are the strain and stress tensor components of the piezoelectric phase along the length direction, $E_{3}$ and $D_{3}$ are the vector components of the electric field and electric displacement, ${ }^{p} S_{33}$ and ${ }^{p} d_{33}$ are the compliance and piezoelectric coefficients, and ${ }^{p} \varepsilon_{33}$ is the dielectric permittivity. The magnetostrictive phase can be described by the following equations:

$$
{ }^{m} S_{3}={ }^{m} s_{33}{ }^{m} T_{3}+{ }^{m} q_{33} H_{3}, \quad B_{3}={ }^{m} q_{33}{ }^{m} T_{3}+{ }^{m} \mu_{33} H_{3},
$$

where ${ }^{m} S_{3}$ and ${ }^{m} T_{3}$ are the strain and stress tensor components of the piezomagnetic phase along the length direction, $H_{3}$ and $B_{3}$ are the vector components of the magnetic field and magnetic flux induction, ${ }^{m} S_{33}$ and ${ }^{m} q_{33}$ are the compliance and piezomagnetic coefficients, and ${ }^{m} \mu_{33}$ is the magnetic permittivity. Equations (1) and (2) were then solved under the following boundary conditions:

$$
\begin{gathered}
{ }^{m} S_{3}={ }^{p} S_{3}, \\
v^{p} T_{3}=-(1-v)^{m} T_{3},
\end{gathered}
$$

where $v=\frac{t_{p}}{t_{p}+2 t_{m}}$ denotes the thickness fraction of the piezoelectric phase.

Using open circuit conditions $\left(H_{3}=0\right)$, the following relationship for $\alpha_{\mathrm{B}}$ can be obtained

$$
\alpha_{B}=\left|\frac{d B_{3}}{d V_{3}}\right|=\left|\frac{v^{p} d_{33}{ }^{m} q_{33}}{(1-v)^{p} s_{33}+v^{m} s_{33}}\right| .
$$

From Eq. (5), the value of $\alpha_{\mathrm{B}}$ can be calculated as a function of $v$ for a Metglas/PZT/Metglas in a multi-push-pull configuration, 


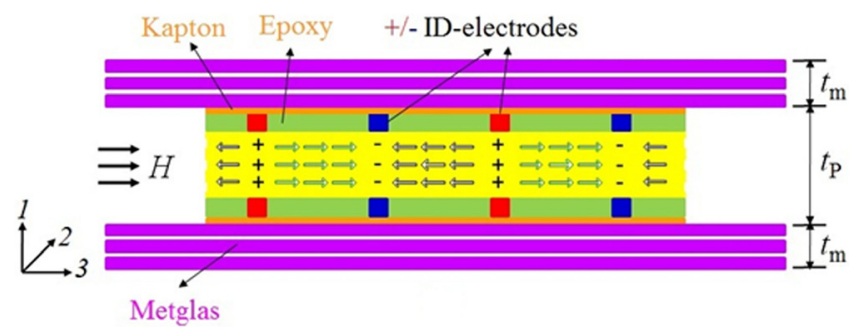

FIG. 1. Schematic diagram of simplified Metglas/PZT/Metglas multi-L-L structure laminate consisting of a Kapton/piezofiber core composite and $\mathrm{N}$ layers Metglas on the bottom and top of the core composite, where the polarization of piezofibers was idealized to be arranged in the longitudinal direction.

as shown in Fig. 2. The following parameters were used in the calculations: ${ }^{9,14}{ }^{p} d_{33}=440 \times 10^{-12} \mathrm{C} / \mathrm{N},{ }^{m} q_{33}=50.3 \times 10^{-9}$ $\mathrm{m} / \mathrm{A},{ }^{p} s_{33}=17.3 \times 10^{-12} \mathrm{~m}^{2} / \mathrm{N}$, and ${ }^{m} s_{33}=10 \times 10^{-12} \mathrm{~m}^{2} / \mathrm{N}$. In this figure, it can be seen that the predicted values of $\alpha_{\mathrm{B}}$ for the multi-push-pull mode are much higher than those for the L$\mathrm{T}$ one. Furthermore, the predicted values of $\alpha_{\mathrm{B}}$ for Terfenol-D/ PMN-PT, or other material couples, L-T mode laminates are quite low, which agrees well with previously reported experimental values. ${ }^{10,12}$ Accordingly, the CME effect in laminates having a multi-push-pull configuration should be experimentally investigated.

To confirm this prediction, a Metglas/PZT/Metglas multi-push-pull mode laminate was fabricated. A $40 \mathrm{~mm} \times 10 \mathrm{~mm}$ PZT bundle served as the core of the laminates, which consisted of five $40 \mathrm{~mm} \times 2 \mathrm{~mm}$ PZT-5A fibers (Smart Material Corp., Sarasota, FL) that were oriented along the length direction of the laminates. Two interdigitated Kapton electrodes were bonded to the top and bottom surfaces of the piezoelectric bundle with epoxy resin (Stycast 1264, USA). $N$ layers of $80 \mathrm{~mm} \times 10 \mathrm{~mm} \mathrm{Fe}_{74.4} \mathrm{Co}_{21.6}$ $\mathrm{Si}_{0.5} \mathrm{~B}_{3.3} \mathrm{Mn}_{0.1} \mathrm{C}_{0.1}$ Metglas foils (Vitrovac 7600F, Hanau, German) were then laminated to both the top and bottom surfaces of the interdigitated electrode/PZT core composite,

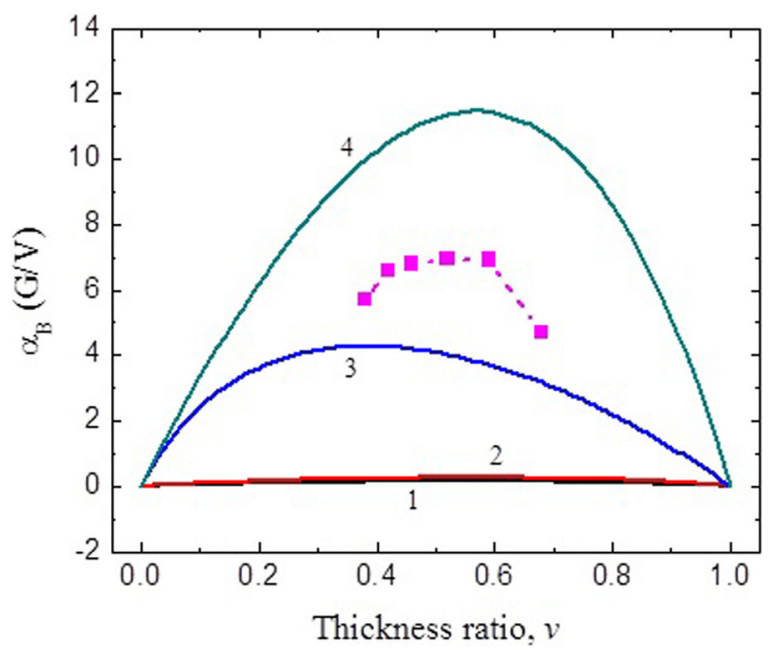

FIG. 2. $\alpha_{\mathrm{B}}$ as a function of the thickness ratio $v$. Lines 1,2 , and 3 represent the calculations for Terfenol-D/PMN-PT, Metglas/PMN-PT and Metglas/ PZT L-T mode laminates using Refs. 10 and 12, with the data in these paper respectively. Lines 4 are calculations for Metglas/PZT/Metglas multi-pushpull mode laminates using Eq. (5). Dots are the experimental data.

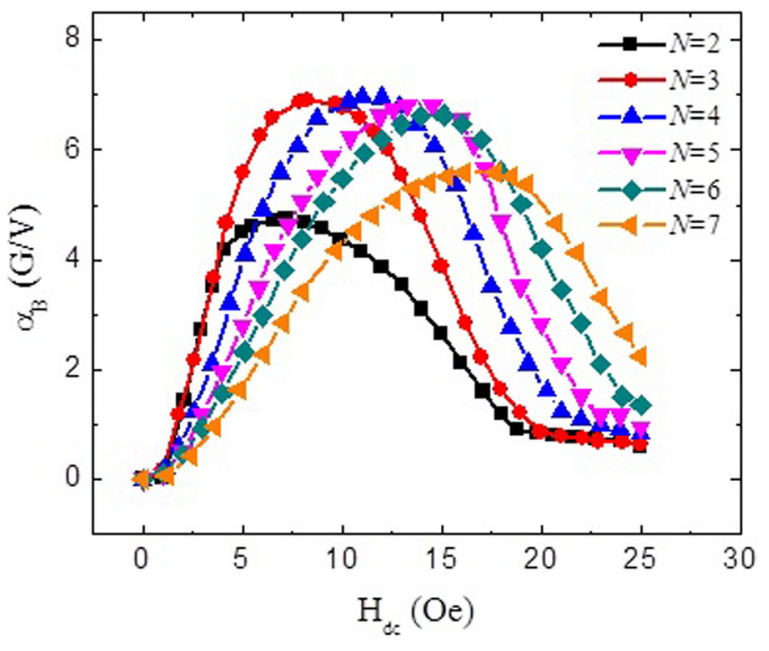

FIG. 3. The value of $\alpha_{\mathrm{B}}$ as a function of $\mathrm{H}_{\mathrm{dc}}$ for Metglas/PZT/Metglas laminates with different $N$ at an applied ac voltage of $50 \mathrm{~V}$ at the frequency of $1 \mathrm{kHz}$.

with a different epoxy resin (West System 105/206, USA). More detailed information of lamination process can be found in Ref. 16. Laminates with different numbers $N$ of Metglas layers bonded to both sides of the PZT core composite were fabricated for $N=2$ to 7 .

To measure the magnetic induction $\left(\mathrm{B}_{3}\right)$, a search coil was wrapped around the laminate. A signal from a lock-in amplifier (SR-850) was amplified by a high voltage amplifier, which was then applied as a voltage $\left(\mathrm{V}_{3}\right)$ across the PZT fibers. A change of the magnetic flux in the Metglas resulted, which was measured as an induced voltage in the search coils that was then subsequently measured by the lock-in amplifier. Figure 3 shows the value of $\alpha_{B}$ as a function of $H_{d c}$ for laminates with different numbers of layers epoxied to both sides of the core composite $(f=1 \mathrm{kHz})$. It can be seen that $\alpha_{\mathrm{B}}$ increased as $\mathrm{H}_{\mathrm{dc}}$ was increased, reached a maximum value, and subsequently decreased as $\mathrm{H}_{\mathrm{dc}}$ was further increased. Furthermore, it can be seen that the maximum value of $\alpha_{\mathrm{B}}$ increased with increasing number of Metglas layers $N$, and then decreased with further increase in $N$. The maximum value of $\alpha_{\mathrm{B}}$ was $6.94 \mathrm{G} / \mathrm{V}$ under $\mathrm{H}_{\mathrm{dc}}=11$ Oe for

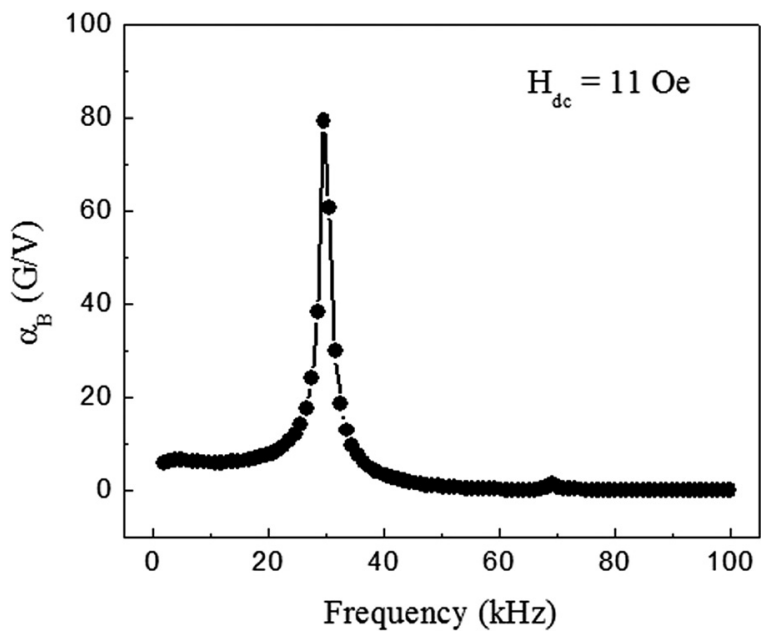

FIG. 4. The frequency response of $\alpha_{\mathrm{B}}$ at an applied ac voltage of $50 \mathrm{~V}$ and a $\mathrm{H}_{\mathrm{dc}}$ of $11 \mathrm{Oe}$. 
$N=4(v=0.52)$. The dependence of the values of $\alpha_{\mathrm{B}}$ on $N$ can be attributed to the differences of effective strain on the Metglas layers. ${ }^{15}$ Using an optimum $v$, the effective strain transferred from PZT fibers to Metglas is larger. Thus, the larger change in the induced magnetization leads to higher values of $\alpha_{\mathrm{B}}$, giving rise to a maximum CME response for $N=4$.

The maximum values of $\alpha_{\mathrm{B}}$ are shown in Fig. 2, as a function of the thickness ratio $v$, calculated from $N$. The experimental values of $\alpha_{\mathrm{B}}$ exhibited similar trends to the predicted ones. However, due to a nonideal interfacial bonding (i.e., coupling factor $0<\mathrm{k}<1$ ) between the piezoelectric and magnetostrictive phases, ${ }^{13}$ the experimental values were only $60 \%$ of the theoretical ones. This strongly suggests that there remains notable potential to enhance the value of $\alpha_{B}$ by improving the interfacial bonding conditions. The frequency dependence of $\alpha_{\mathrm{B}}$ for the laminate with $N=4$ is given in Fig. 4 , for $\mathrm{H}_{\mathrm{dc}}=11 \mathrm{Oe}$. The maximum value of $\alpha_{\mathrm{B}}$ was $79.5 \mathrm{G} / \mathrm{V}$ at an electromechanical resonance (EMR) frequency of 29.6 $\mathrm{kHz}$. Such giant values of $\alpha_{\mathrm{B}}$ at EMR are $>25 \times$ higher than those previously reported for other modes. ${ }^{12}$

In summary, a giant $\mathrm{CME}$ effect has been predicted and measured for Metglas/PZT/Metglas multi-push-pull mode laminates. The results demonstrated that these laminates possess a value of $\alpha_{B}=6.94 \mathrm{G} / \mathrm{V}$ at an optimum thickness ratio of $v=0.52(f=1 \mathrm{kHz})$ under $\mathrm{H}_{\mathrm{dc}}=11$ Oe. Furthermore, a giant value of $\alpha_{\mathrm{B}}=79.5 \mathrm{G} / \mathrm{V}$ was found at the EMR.

${ }^{1}$ N. A. Spaldin and M. Fiebig, Science 309(5733), 391 (2005).

${ }^{2}$ C.-W. Nan, M. I. Bichurin, S. Dong, D. Viehland, and G. Srinivasan, J. Appl. Phys. 103(3), 031101 (2008).

${ }^{3}$ W. Eerenstein, N. D. Mathur, and J. F. Scott, Nature 442(7104), 759 (2006).

${ }^{4}$ W. Eerenstein, M. Wiora, J. L. Prieto, J. F. Scott, and N. D. Mathur, Nature Mater. 6(5), 348 (2007).

${ }^{5}$ R. Ramesh and N. A. Spaldin, Nature Mater. 6, 21 (2007).

${ }^{6}$ S. Dong, J. Zhai, J. Li, and D. Viehland, Appl. Phys. Lett. 89(25), 252904 (2006).

${ }^{7}$ J. Zhai, Z. Xing, S. Dong, J. Li, and D. Viehland, J. Am. Ceram. Soc. 91(2), 351 (2008).

${ }^{8}$ J. Ma, J. Hu, Z. Li, and C. W. Nan, Adv. Mater. 23(9), 1062 (2011).

${ }^{9}$ Y. Wang, D. Gray, D. Berry, J. Gao, M. Li, J. Li, and D. Viehland, Adv. Mater. 23(35), 4111 (2011).

${ }^{10}$ Y. Jia, S. W. Or, H. L. W. Chan, X. Zhao, and H. Luo, Appl. Phys. Lett. $\mathbf{8 8}(24), 242902$ (2006).

${ }^{11}$ Y. Wang, F. Wang, S. W. Or, H. L. W. Chan, X. Zhao, and H. Luo, Appl. Phys. Lett. 93(11), 113503 (2008).

${ }^{12}$ H. C. Xuan, L. Y. Wang, S. C. Ma, Y. X. Zheng, Q. Q. Cao, D. H. Wang, and Y. W. Du, Appl. Phys. Lett. 98(5), 052505 (2011).

${ }^{13}$ M. Bichurin, V. Petrov, and G. Srinivasan, Phys. Rev. B 68(5), 054402 (2003).

${ }^{14}$ F. Fang, C. P. Zhao, and W. Yang, Sci. China, Ser. G 54(4), 581 (2011).

${ }^{15}$ J. Das, J. Gao, Z. Xing, J. F. Li, and D. Viehland, Appl. Phys. Lett. 95(9), 092501 (2009).

${ }^{16}$ M. H. Li, D. Berry, J. Das, D. Gray, J. F. Li, and D. Viehland, J. Am. Ceram. Soc. 94(11), 3738 (2011). 